Preprint YERPHI-1448(18)-95

\title{
HIGGS BOSON MASSES IN SUPERSYMMETRIC THEORIES
}

\author{
ASATRIAN H.M., YEGHIYAN G. K. \\ Yerevan Physics Institute, Alikhanyan Br. 2, Yerevan, Armenia \\ e-mail: "hrach@uniphi.yerphi.am"
}

\begin{abstract}
The Higgs boson mass problem is considered in the next to minimal supersymmetric standard model. The Higgs potential and the renormalization group equations for the gauge, Yukawa and scalar coupling constants are analyzed. The restrictions for the Higgs boson masses are found for the cases of presence and absence of spontaneous $\mathrm{CP}$ - violation.
\end{abstract}

\section{Yerevan Physics Institute Yerevan 1995}


1.The aim of our paper is to consider the problem of Higgs masses in the next to minimal supersymmetric standard model (NMSSM). Such a model contains an additional Higgs singlet, as compared with minimal supersymmetric standard model (MSSM). The cause of the consideration of the NMSSM was connected, in particular, with desire to avoid the explicit mass term for the Higgs doublets in the superpotential [1]. On the other hand it is interesting to verify, if the predictions of MSSM remain valid for the more general supersymmetric models. The other interesting problem is connected with CP -violation: it is known [2] that in MSSM one can't realize the realistic scenario of spontaneous CP-violation, therefore it is natural to consider, if this scenario possible in NMSSM [3]. The spontaneous CP-violation leads, in particular, to the existence of large (compared with the standard model predictions) electric dipole moment of fermions [3, 4, 5].

2.We will investigate the restrictions for Higgs boson masses which can be obtained from the Higgs potential and renormalization group equations in NMSSM. The restrictions for Higgs boson masses in NMSSM was investigated in [6, 7, 8, 9] for the case of absence of spontaneous CP violation. Here the case of the spontaneous CP- violation will be also considered. The problem of possible restrictions which can be obtained for particles masses from the renormalization group equations analysis in the case of (nonsupersymmetric) standard model also was considered in literature. There various approaches have been developed [10, 11, 12]. Such a investigations have been carried out also for the SUSY extensions of the standard model (see 13 and references therein).

In our consideration we assume that all of the (gauge, Yukawa, scalar) coupling constants of our theory are small between weak $(\sim 100 \mathrm{GeV})$ and unification scales $\left(\sim\left(10^{16}-10^{18}\right) \mathrm{GeV}\right)$, so that the perturbation theory applies [11, 14]. Besides this, for the theory to be a correct one, the condition of vacuum stability is necessary. These conditions leads to restrictions for parameters of the scalar potential. As a result, one obtains some bounds for Higgs particles masses.

3.Let as proceed to description of our model. The Higgs sector consists of two Higgs doublets:

$$
H_{1}=\left(\begin{array}{c}
\xi_{1}^{+} \\
\xi_{1}^{o}
\end{array}\right) \quad H_{2}=\left(\begin{array}{c}
\xi_{2}^{o} \\
\xi_{2}^{-}
\end{array}\right)
$$

with hypercharges $Y\left(H_{1}\right)=1, Y\left(H_{2}\right)=-1$ and the complex $S U(2)_{L} \times U(1)_{Y}$ 
singlet $\mathrm{N}$. The superpotential for one quark generation is the following [5]:

$$
W=\frac{\lambda_{1}}{3} N^{3}+\lambda_{2} N H_{1} H_{2}+h_{u} H_{2} Q_{L}^{c} u_{R}+h_{d} H_{1} Q_{L}^{c} d_{R}
$$

where

$$
Q_{L}=\left(\begin{array}{c}
u_{L} \\
d_{L}
\end{array}\right) \quad Q_{L}^{c}=i \sigma_{2} Q_{L}^{*}
$$

The scalar potential is given by:

$$
V=\Sigma\left|\frac{\partial W}{\partial \varphi_{i}}\right|^{2}+\frac{1}{2} \Sigma\left|g_{a} \varphi_{i}^{+} T_{a} \varphi i\right|^{2}+V_{\text {soft }}
$$

where $\varphi_{i}(\mathrm{i}=1,2 \ldots)$ are the scalar components of all superfields, $g_{r}$ and $T_{r}(\mathrm{r}=1,2,3)$ are coupling constants and generators of gauge groups $U(1)_{Y}$, $S U(2)_{L}, S U(3)_{c}$ respectively [15, 16]. The term $V_{\text {soft }}$ is the part of potential which breaks the supersymmetry. It is usually assumed that supersymmetry breaking occurs in the hidden sector of $\mathrm{N}=1$ supergravity theories [17, 18, 19, 20]. For the simplest $\mathrm{N}=1$ supergravity models the structure of supersymmetry breaking terms is:

$$
V_{\text {soft }}=A m W_{3}+B m W_{2}+m^{2} \Sigma\left|\varphi_{i}\right|^{2}+M \lambda_{r} \lambda_{r}+\text { h.c. }
$$

where $\mathrm{m}$ is the gravitino mass, $\mathrm{A}, \mathrm{B}$ are supersymmetry breaking parameters, $\lambda_{r}(\mathrm{r}=1,2,3)$ are gaugino fields and $\mathrm{M}$ is gauginos universal mass.

In the present paper we will consider more general supergravity models [3, 21] to obtain more general restrictions for the Higgs masses in supersymmetric theories. On the other hand such a general consideration allows one to explain observable $\mathrm{CP}$ violating effects only by spontaneous $\mathrm{CP}$ - violation [3]. For such a models the supersymmetry breaking term is the following:

$$
\begin{aligned}
V_{\text {soft }} & =m_{1}^{2}\left|H_{1}\right|^{2}+m_{2}^{2}\left|H_{2}\right|^{2}+m_{3}^{2}|N|^{2}+ \\
& +\left(m_{4} N H_{1} H_{2}+\frac{m_{5}}{3} N^{3}+\text { h.c. }\right)+\ldots
\end{aligned}
$$

where we omit terms connected with sfermion and gaugino fields and write only terms connected with Higgs fields. It is necessary to note that unlike of the ordinary case (3) in (4) the supersymmetry breaking terms are not universal. We want to stress that usually it is assumed that supersymmetry 
breaking terms given by (3) or (4) for the MSSM and NMSSM respectively have the given form for unification energies $M_{G} \sim\left(10^{16}-10^{18}\right) \mathrm{GeV}$. In MSSM below this scale due to different interactions of Higgs doublets with fermions the mass terms of $H_{1}$ and $H_{2}$ differ from each other. For the considering case, although the values of the supersymmetry breaking parameters are changing, when, evaluating from unification energies to the $M_{s}$, the mass terms for Higgs doublet are different from the beginning and the form of supersymmetry breaking terms (4) remains the same. This means that we can consider that this form is valid for the supersymmetry breaking scale also.

From (1), (2), (4) one obtains the scalar potential for the Higgs fields $H_{1}$, $\mathrm{H}_{2}, \mathrm{~N}$ :

$$
\begin{aligned}
V & =\frac{g_{2}^{2}+g_{1}^{2}}{8}\left(\left(H_{1}^{+} H_{1}\right)^{2}+\left(H_{2}^{+} H_{2}\right)^{2}\right)+\frac{g_{2}^{2}-g_{1}^{2}}{4}\left|H_{1}\right|^{2}\left|H_{2}\right|^{2}+\left(\lambda_{2}^{2}-\frac{1}{2} g_{2}^{2}\right)\left|H_{1} H_{2}\right|^{2} \\
& +\lambda_{2}^{2}|N|^{2}\left(\left|H_{1}\right|^{2}+\left|H_{2}\right|^{2}\right)+\lambda_{1} \lambda_{2}\left(\left(N^{2}\right)^{+} H_{1} H_{2}+h . c .\right)+\lambda_{1}^{2}|N|^{4}+ \\
& +m_{4}\left(N H_{1} H_{2}+\text { h.c. }\right)+\frac{m_{5}}{3}\left(N^{3}+h . c\right)+m_{1}^{2}\left|H_{1}\right|^{2}+m_{2}^{2}\left|H_{2}\right|^{2}+m_{3}^{2}|N|^{2}
\end{aligned}
$$

The potential (5) is positively defined for large values of the Higgs fields $H_{1}, H_{2}, N$ vacuum expectation values (VEV) and there is no problem with vacuum stability in (5).

In general, the supersymmetry and electroweak symmetry breaking scales are different. This means that the scalar potential at electroweak scale $(\sim$ $100 \mathrm{GeV}$ ) has the form, different from (5). It is given by

$$
\begin{aligned}
V & =\frac{1}{2} a_{1}\left(H_{1}^{+} H_{1}\right)^{2}+\frac{1}{2} a_{2}\left(H_{2}^{+} H_{2}\right)^{2}+a_{3}\left|H_{1}\right|^{2}\left|H_{2}\right|^{2}+a_{4}\left|H_{1} H_{2}\right|^{2}+a_{5}|N|^{2}\left|H_{1}\right|^{2} \\
& +a_{6}|N|^{2}\left|H_{2}\right|^{2}+a_{7}\left(\left(N^{2}\right)^{+} H_{1} H_{2}+h . c .\right)+a_{8}|N|^{4}++m_{4}\left(N H_{1} H_{2}\right. \\
& + \text { h.c. })+\frac{m_{5}}{3}\left(N^{3}+h . c\right)+m_{1}^{2}\left|H_{1}\right|^{2}+m_{2}^{2}\left|H_{2}\right|^{2}+m_{3}^{2}|N|^{2}
\end{aligned}
$$

It is easily to see that the potential (6) contains the same terms as (5). The difference is only that the coupling constants in (6) are not connected by the relations which come from supersymmetry. The behavior with the energy of the coupling constants $a_{i}(i=1,2, \ldots, 8)$ (and also for the Yukawa and gauge couplings) is given by renormalization group equations. The parameters of potentials (5) and (6) are connected by the relations:

$$
\begin{aligned}
& a_{5}=a_{6}=\lambda_{2}^{2}, \quad a_{7}=\lambda_{1} \lambda_{2}, \quad a_{8}=\lambda_{1}^{2} \\
& a_{1}=a_{2}=\frac{1}{4}\left(g_{1}^{2}+g_{2}^{2}\right), \quad a_{3}=\frac{1}{4}\left(g_{2}^{2}-g_{1}^{2}\right), \quad a_{4}=\lambda_{2}^{2}-\frac{1}{2} g_{2}^{2}
\end{aligned}
$$


The relations (7) are valid at the supersymmetry breaking scale $M_{s}$ and higher.

4.In our model the electroweak symmetry breaking takes place, when the Higgs fields $H_{1}, H_{2}, N$ in potential (6) develop nonzero VEV's. To provide the electric charge conservation we shall chose these VEV's in the following form:

$$
<H_{1}>=\left(\begin{array}{c}
0 \\
v_{1}
\end{array}\right) \quad<H_{2}>=\left(\begin{array}{c}
v_{2} e^{i \varphi} \\
0
\end{array}\right) \quad<N>=v_{3} e^{i \alpha}
$$

For Higgs VEV's given by (9) the potential (7) is given by:

$$
\begin{aligned}
V_{o}\left(v_{1}, v_{2}, v_{3}\right) & =\frac{1}{2} a_{1} v_{1}^{4}+\frac{1}{2} a_{2} v_{2}^{4}+\left(a_{3}+a_{4}\right) v_{1}^{2} v_{2}^{2}+a_{5} v_{1}^{2} v_{3}^{2}+a_{6} v_{2}^{2} v_{3}^{2} \\
& +2 a_{7} v_{1} v_{2} v_{3}^{2} \cos (\varphi-2 \alpha)+2 m_{4} v_{1} v_{2} v_{3} \cos (\varphi+\alpha)+ \\
& +\frac{2}{3} m_{5} v_{3}^{3} \cos 3 \alpha+a_{8} v_{3}^{4}+m_{1}^{2} v_{1}^{2}+m_{2}^{2} v_{2}^{2}+m_{3}^{2} v_{3}^{2}
\end{aligned}
$$

It is easily to see that the potential (9) is invariant for discrete transformations of the type

$$
\varphi \rightarrow \varphi+\frac{\pi}{3}, \quad \alpha \rightarrow \alpha+\frac{2 \pi}{3}, \quad m_{4} \rightarrow-m_{4}, \quad a_{7} \rightarrow-a_{7}
$$

This means that we can choose (without loss of generality)

$$
v_{1}, v_{2}, v_{3}>0 ; \quad-\frac{\pi}{2} \leq \varphi \leq \frac{\pi}{2} ; \quad-\frac{\pi}{6} \leq \alpha \leq \frac{\pi}{6}
$$

For the potential to be a stable one (this means that for the large values of $v_{i}, i=1.2 .3$ it must be positive) the following conditions must be satisfied:

$$
\begin{aligned}
a_{1}>0, \quad a_{2}>0, \quad a_{8} & >0 \\
a_{3}+a_{4}+\sqrt{a_{1} a_{2}} & >0 \\
a_{6}+\sqrt{2 a_{1} a_{8}}>0, \quad a_{6}+\sqrt{2 a_{2} a_{8}} & >0
\end{aligned}
$$

The equations for the potential minimum have the form

$$
\begin{array}{r}
\frac{\partial V}{\partial \varphi}=\frac{\partial V}{\partial \alpha}=0 \\
\frac{\partial V}{\partial v_{1}}=\frac{\partial V}{\partial v_{2}}=\frac{\partial V}{\partial v_{3}}=0
\end{array}
$$


The first two conditions can be written in the form:

$$
\begin{gathered}
m_{4} \sin (\varphi+\alpha)+a_{7} v_{3} \sin (\varphi-2 \alpha)=0 \\
2 a_{7} v_{1} v_{2} v_{3} \sin (2 \alpha-\varphi)+m_{4} v_{1} v_{2} \sin (\alpha+\varphi)+m_{5} v_{3}^{2} \sin 3 \alpha=0
\end{gathered}
$$

The equations (12), (13) have two type of solutions. First, with $\alpha=\varphi=0$ corresponds to the case of absence of spontaneous CP-violation. The second type of solution with nonzero phases $\alpha, \varphi$ corresponds to the case of spontaneous CP-violation. For equations (12), (13) to have nonzero solutions for $\varphi$ and $\alpha$ the following condition must be satisfied:

$$
3 a_{7} v_{1} v_{2} \cos \varphi+\frac{v_{3} m_{5}}{m_{4}}\left(a_{7} v_{3} \cos 2 \alpha+m_{4} \cos \alpha\right)=0
$$

Three last conditions of (11) can be written in the following form:

$$
\begin{aligned}
& a_{1} v_{1}^{3}+\left(a_{3}+a_{4}\right) v_{1} v_{2}^{2}+a_{5} v_{1} v_{3}^{2}+m_{1}^{2} v_{1} \\
& +\quad\left(m_{4} \cos (\varphi+\alpha)+a_{7} v_{3} \cos (\varphi-2 \alpha)\right) v_{3} v_{2}=0 \\
& a_{2} v_{2}^{3}+\left(a_{3}+a_{4}\right) v_{2} v_{1}^{2}+a_{6} v_{2} v_{3}^{2}+m_{2}^{2} v_{2}+ \\
& +\quad\left(m_{4} \cos (\varphi+\alpha)+a_{7} v_{3} \cos (\varphi-2 \alpha)\right) v_{3} v_{1}=0 \\
& 2 a_{8} v_{3}^{3}+\left(a_{5} v_{1}^{2}+a_{6} v_{2}^{2}+2 a_{7} v_{1} v_{2} \cos (\varphi-2 \alpha)\right) v_{3} \\
& +m_{5} v_{3}^{2} \cos 3 \alpha+m_{4} v_{1} v_{2} \cos (\varphi+\alpha)+m_{3}^{2} v_{3}=0
\end{aligned}
$$

The equations (15)-(17) are satisfied for $v_{1}=v_{2}=v_{3}=0$. We are looking for solutions with

$$
v_{1} \neq 0, v_{2} \neq 0, v_{3} \neq 0 \text {. }
$$

For the solutions (18) to be a real minimum of the potential the value of $V_{o}\left(v_{1}, v_{2}, v_{3}\right)$ (where $v_{1}, v_{2}, v_{3}$ are nonzero solutions of equations (12), (13), (15)-(17)) must be negative:

$$
\begin{aligned}
V\left(v_{1}, v_{2}, v_{3}\right) & =\frac{1}{2}\left(\frac{1}{3} m_{5} v_{3}^{3} \cos 3 \alpha+m_{4} v_{1} v_{2} v_{3} \cos (\varphi+\alpha)+m_{1}^{2} v_{1}^{2}\right. \\
& \left.+m_{2}^{2} v_{2}^{2}+m_{3}^{2} v_{3}^{2}\right)<V(0,0,0)=0
\end{aligned}
$$

We must take into account also the following: for the solution with nonzero values of $\alpha, \varphi$ and spontaneous CP-violation to be the physical minimum of 
the potential the potential value in this point must be less than its value for $\alpha=0, \varphi=0$. This means that the following condition must be satisfied:

$$
m_{5} \sin \frac{\varphi+\alpha}{2} \sin \frac{\varphi-2 \alpha}{2}<0
$$

5.Let us consider now the masses of Higgs particles. After the spontaneous breaking of electroweak symmetry it appears five neutral and one complex charged fields. The goldstone mode for neutral fields can be excluded by the transformation:

$$
\begin{aligned}
\xi_{1}^{o} & =v_{1}+\frac{1}{\sqrt{2}}\left(\Phi_{1} \cos \beta-\Phi_{2} \sin \beta-i(G \cos \beta-A \sin \beta)\right) \\
\xi_{2}^{o} & =e^{i \varphi}\left(v_{2}+\frac{1}{\sqrt{2}}\left(\Phi_{1} \sin \beta+\Phi_{2} \cos \beta+i(G \sin \beta+A \cos \beta)\right)\right) \\
N & =e^{i \alpha}\left(N_{1}+i N_{2}\right)
\end{aligned}
$$

where $\Phi_{1}, \Phi_{2}, A, N_{1}, N_{2}$ are new neutral fields, $\mathrm{G}$ is the goldstone mode, $\tan \beta=\frac{v_{2}}{v_{1}}$. The $5 \times 5$ symmetric mass matrix $M_{i j}^{2}, i, j=1, \ldots, 5$ for fields $\Phi_{1}, \Phi_{2}, A, N_{1}, N_{2}$ for the case of the presence of CP- violation is given by formula (A1) of the Appendix A. For the case of absence of CP- violation the mass matrix has more simple form: it consists of two matrices, one of them $3 \times 3$ is the mass matrix of CP- even fields $\Phi_{1}, \Phi_{2}, N_{1}$, the second one is $2 \times 2$ mass matrix of $\mathrm{CP}$ - odd fields $A, N_{2}$. They are given by formulae (A2) and (A3) of the Appendix A. For the charged Higgs particles one can exclude the Goldstone mode by the transformation:

$$
\begin{gathered}
\xi_{1}^{+}=-G^{+} \cos \beta+e^{i \varphi} H^{+} \sin \beta \\
\xi_{2}^{-}=e^{i \varphi} G^{-} \sin \beta+H^{-} \cos \beta
\end{gathered}
$$

The mass of charged Higgs is given by:

$$
m_{H^{+}}^{2}=-\frac{2\left(m_{4} \cos (\varphi+\alpha)+a_{7} v_{3} \cos (\varphi-2 \alpha)\right) v_{3}}{\sin 2 \beta}-a_{4} \eta^{2}
$$

where

$$
\eta^{2}=v_{1}^{2}+v_{2}^{2}=(174 G e V)^{2}
$$

It is necessary to note that the condition of positiveness of charged Higgs mass squared provides the conservation of electric charge i.e. the Higgs boson VEV's have the form (8) [9] 
6.To obtain the bounds for masses of the Higgs particles one must investigate the possible restrictions for parameters of Higgs potential (6). The restrictions for the coupling constants $a_{i}, i=1, \ldots 8$ one can obtain, by analyzing the renormalization group equations for $a_{i}, i=1, \ldots 8$, gauge $g_{r}, r=1,2,3$ and t and b quark Yukawa couplings $h_{t}=\frac{m_{t}}{v_{2}}, h_{b}=\frac{m_{b}}{v_{1}}$ (one can neglect other Yukawa couplings because of their smallness with regard to $h_{t}$ and $h_{b}$ ).

In the region between the electroweak symmetry breaking scale and supersymmetry breaking scale they have the form [6, 22] $\left(t=\ln \left(\frac{Q}{100 \mathrm{GeV}}\right)\right.$ :

$$
\begin{aligned}
16 \pi^{2} \frac{\partial a_{i}}{\partial t} & =f_{i}, i=1, \ldots 8 \\
16 \pi^{2} \frac{\partial h_{t}}{\partial t} & =h_{t}\left(\frac{9}{2} h_{t}^{2}+\frac{1}{2} h_{b}^{2}-\frac{17}{12} g_{1}^{2}-\frac{9}{4} g_{2}^{2}-8 g_{3}^{2}\right) \\
16 \pi^{2} \frac{\partial h_{b}}{\partial t} & =h_{b}\left(\frac{9}{2} h_{b}^{2}+\frac{1}{2} h_{t}^{2}-\frac{5}{12} g_{1}^{2}-\frac{9}{4} g_{2}^{2}-8 g_{3}^{2}\right) \\
16 \pi^{2} \frac{\partial g_{i}}{\partial t} & =-c_{i} g_{i}^{3}
\end{aligned}
$$

where

$$
c_{1}=-7, \quad c_{2}=3, \quad c_{3}=7
$$

and $f_{i}, \mathrm{i}=1, \ldots, 8$ are given by the formula $(\mathrm{B} 1)$ of the Appendix B. The values of gauge constants $g_{r}(\mathrm{r}=1,2,3)$ at electroweak breaking scale $\sim M_{Z}$ are the following [13]:

$$
g_{1}=0.358 \quad g_{2}=0.651 \quad g_{3}=1.218
$$

Above the supersymmetry breaking scale some of the constants $a_{i}(\mathrm{i}=1, \ldots 8)$ are unified according to equations (7), and the renormalization group equations should be written for the quantities $\lambda_{1}, \lambda_{2}, h_{t}, h_{b}, g_{r}$ instead of the quantities $a_{i}, h_{t}, h_{b}, g_{r}(\mathrm{r}=1,2,3 ; \mathrm{i}=1, \ldots 8)$. These equations are as follows [23] $\left(t=\ln \frac{Q}{M_{s}}\right)$ :

$$
\begin{aligned}
16 \pi^{2} \frac{d h_{t}}{d t} & =h_{t}\left(6 h_{t}^{2}+h_{b}^{2}+\lambda_{2}^{2}-\frac{13}{9} g_{1}^{2}-3 g_{2}^{2}-\frac{16}{3} g_{3}^{2}\right) \\
16 \pi^{2} \frac{d h_{b}}{d t} & =h_{b}\left(6 h_{b}^{2}+h_{t}^{2}+\lambda_{2}^{2}-\frac{10}{9} g_{1}^{2}-3 g_{2}^{2}-\frac{16}{3} g_{3}^{2}\right) \\
16 \pi^{2} \frac{d \lambda_{2}}{d t} & =\lambda_{2}\left(2 \lambda_{1}^{2}+4 \lambda_{2}^{2}+3\left(h_{t}^{2}+h_{b}^{2}\right)-g_{1}^{2}-3 g_{2}^{2}\right) \\
16 \pi^{2} \frac{d \lambda_{1}}{d t} & =6 \lambda_{1}\left(\lambda_{1}^{2}+\lambda_{2}^{2}\right)
\end{aligned}
$$




$$
16 \pi^{2} \frac{d g_{i}}{d t}=-c_{i} g_{i}^{3} ; \quad c_{1}=-11, \quad c_{1}=-1, \quad c_{3}=3
$$

To obtain the restrictions for $a_{i}$ at scale $Q=M_{Z}$ we investigate numerically the differential equations (25) from $Q=M_{Z}$ to $Q=M_{s}$ and (26) from $Q=M_{s}$ to $Q=M_{G}$. These restrictions for $a_{i}$ arise in the following way. We require that in the region from $M_{Z}$ to $M_{s}$ and from $M_{s}$ to $M_{G}$ all of the coupling constants must be small so that the perturbation theory applies. This will give some restrictions for values of scalar coupling constants $a_{i}$ at $Q=M_{Z}$ and so for the Higgs masses. One must take into account also the vacuum stability condition which will give an additional restrictions for $a_{i}$.

More precisely, the perturbation theory to be applied, the quantities $a_{i}(i=1, \ldots 8), h_{t}, h_{b}$ at $Q=M_{Z}$ must satisfy the following conditions:

$$
\frac{h_{t}^{2}}{4 \pi}, \frac{h_{b}^{2}}{4 \pi}, \frac{\left|a_{i}\right|}{4 \pi}<1
$$

The conditions (27) must be valid for also for $M_{Z}<Q<M_{s}$. For $M_{s}<Q<$ $M_{G}$, conditions $(27)$ are replaced by the conditions $(\mathrm{k}=1,2)$ :

$$
\frac{\lambda_{k}^{2}}{4 \pi}, \frac{h_{b}^{2}}{4 \pi}, \frac{h_{t}^{2}}{4 \pi}<1
$$

Actually, the conditions (27), (28) are not enough to provide the perturbation theory validity. Severely speaking, the condition of smallness of derivative of coupling constants must be satisfied:

$$
\frac{\partial a_{i}}{\partial t}, \frac{\partial \lambda_{k}}{\partial t}, \frac{\partial h_{t}}{\partial t}, \frac{\partial h_{b}}{\partial t}<A
$$

where $\mathrm{A}$ is a number $\sim 1$.

Let us proceed to concrete results. We will consider that the supersymmetry breaking scale $M_{s}$ is between $100 \mathrm{GeV}$ and $10000 \mathrm{GeV}$ and the unification scale is between $10^{16} \mathrm{GeV}$ and $10^{18} \mathrm{GeV}$. The values of $\mathrm{t}$ and $\mathrm{b}$ quark masses at scale $M_{Z}$ we take equal to $m_{t}=(175 \pm 15) \mathrm{GeV}, m_{b}=(3.5 \pm 0.5) \mathrm{GeV}$ [24, 25].

We investigate numerically the renormalization group equations, taking into account the condition of the validity of the perturbation theory and the vacuum stability condition. If the supersymmetry breaking scale is $M_{s}=$ $M_{Z}$, one obtains the restrictions for $\lambda_{1}, \lambda_{2}, h_{t}$ and $h_{b}$ :

$$
\left|\lambda_{1}\right| \leq 0.5, \quad\left|\lambda_{2}\right| \leq 0.6, \quad 1.0 \leq\left|h_{t}\right| \leq 1.1,\left|h_{b}\right| \leq 1.0
$$


For $M_{s}=1000 \mathrm{GeV}$ and $M_{s}=1000 \mathrm{GeV}$ the restrictions for parameters $a_{i}(i=1, \ldots, 8), h_{t}$ and $h_{b}$ are given in Appendix B.

The Higgs masses depend also on mass parameters of the potential and Higgs boson VEV's $v_{1}, v_{2}, v_{3}$. The mass parameters of this potential $m_{4}, m_{5}$, $m_{1}^{2}, m_{2}^{2}, m_{3}^{2}$ are connected with supersymmetry breaking and are of order of supersymmetry breaking scale. The Higgs VEV's $v_{1}, v_{2}$ are restricted by the condition (24). The restrictions for their ratio

$$
\frac{v_{2}}{v_{1}}=\frac{v_{2}}{\sqrt{\eta^{2}-v_{2}^{2}}}=\tan \beta
$$

are obtained from values of $\mathrm{t}$ and b-quark masses. From (31) one obtains for $m_{t}=(175 \pm 15) \mathrm{GeV}, m_{b}=(3.5 \pm 0.5) \mathrm{GeV}$

$$
1<\tan \beta<60
$$

The singlet's VEV $v_{3}$, generally speaking is not connected with the supersymmetry breaking and so its scale is not coincide with the supersymmetry breaking scale $M_{s}$. However the equations of the potential minimum (15)(17) give an additional restrictions for this parameter.

Let as try to analyze qualitatively which bounds one can obtain for neutral and charged Higgs masses, when apply the above obtained restrictions.

First of all let us consider the case of absence of spontaneous CP-breaking.

It is easily to see from the formula (23) and the previous analysis that the charged Higgs particle mass can be as heavy as supersymmetry breaking scale.

As concerned the neutral Higgs masses, as we have mentioned above, the mass matrix consists of two separate $3 \times 3$ and $2 \times 2$ matrices for $\mathrm{CP}$ even and CP odd particles respectively. The analysis of these mass matrix shows that the upper bound of mass of one CP even particle is of order $\eta=174 \mathrm{GeV}$ and four other particles masses, generally speaking, are of order of supersymmetry breaking scale $M_{s}$. This follows, in particular, from the fact that determinant of $\mathrm{CP}$ even particles mass matrix is proportional to $\eta^{2} M_{s}^{4}$ and determinant of $\mathrm{CP}$ odd particle mass matrix is proportional to $M_{s}^{4}$.

The case of the presence of spontaneous CP-violation is more complicated: here one has $5 \times 5$ neutral Higgses mass matrix. In this case the conditions of positiveness of Higgs boson masses are very important. These 
conditions are equivalent to the positiveness of determinant and diagonal minors of matrix (A1). The analytical investigations of these conditions (taking into account also the condition of positiveness of charged Higgs particle) gives the following result: for supersymmetry breaking scale $M_{s} \sim 100 \mathrm{GeV}$ these conditions can't be satisfied simultaneously, i.e for $M_{s} \sim 100 \mathrm{GeV}$ the spontaneous CP breaking can't take place. As concerned $M_{s} \sim 1000 \mathrm{GeV}$ and $M_{s} \sim 10000 \mathrm{GeV}$, the condition of the neutral Higgs mass matrix to be positively determined gives the following inequality which must be satisfied for energies $\sim 100 \mathrm{GeV}$ :

$$
\begin{aligned}
& \max \left(\left(a_{3}+a_{4}-\sqrt{a_{1} a_{2}}\right) \frac{\eta^{2} \sin 2 \beta}{2 v_{3}^{2}}, \frac{a_{7}^{2} \eta^{2} \sin 2 \beta}{2 a_{8} v_{3}^{2}}\right)< \\
& <-a_{7} \frac{\sin 3 \alpha}{\sin (\varphi+\alpha)}<\left(a_{3}+a_{4}+\sqrt{a_{1} a_{2}}\right) \frac{\eta^{2} \sin 2 \beta}{2 v_{3}^{2}}
\end{aligned}
$$

This condition is obtained from requirement that the fourth order diagonal two minors of matrix (A1) which can be obtained, when first or fourth lines and columns are excluded must be positive. Let us note also that for $M_{s} \sim$ $100 \mathrm{GeV}$ the inequalities (33) become the equalities due to the relations (7) and it is not possible to provide the positiveness of the diagonal minors, as we mentioned above. The conditions (33) can be satisfied only due to the fact that the coupling constants $a_{i}$, i=1,..,8, for $M_{s} \sim 1000 \mathrm{GeV}$ or $M_{s} \sim 10000 \mathrm{GeV}$ are not satisfying relations (7) for energies $\sim 100 \mathrm{GeV}$ due to the their evolution from supersymmetry breaking scale to electroweak breaking scale according renormalization group equations (25).

Using the condition (33), we come to the following restrictions for the neutral Higgs particles masses in the case of presence of CP-violation. The lightest neutral Higgs has mass of order of $\eta$ multiplied by the factor of order of radiative corrections to the coupling constants $a_{i}, \mathrm{i}=1, \ldots, 8$, when, evaluating from $1000 \mathrm{GeV}$ or $10000 \mathrm{GeV}$ to $100 \mathrm{GeV}$, i.e., in general, sufficiently small. Two other neutral Higgs boson have masses of order of $\sim \eta$ and masses of two last neutral Higgses can be as heavy as supersymmetry breaking scale $M_{s}$. These results are obtained, in particular, from the fact that due to the condition (33) determinant of matrix (A1) is proportional to $M_{s}^{4} \eta_{6}$ multiplied by the factor(s) of order of radiative corrections to the coupling constants $a_{i}$.

It is interesting to analyze the case, when one or two neutral Higgs particles are almost $S U(2) \times U(1)$ singlets. In this case the situation is the 
following: if both of singlets are much heavier than electroweak breaking scale, then, as it follows from the previous analysis, the remaining neutral scalar particles have a masses of order of $\eta$ or smaller. The following statement is also true: if one of the neutral Higgs boson is much heavier than $\eta$, then it is almost singlet. This means that in all cases one has three neutral visible (nonsinglet) particles with "small" $(\leq \eta)$ masses.

Using the condition (33) and equation (12) one obtains from (23) the following restriction for the mass of charged Higgs particle:

$$
m_{H^{+}}^{2}<\left(a_{3}+\sqrt{a_{1} a_{2}}\right) \eta^{2}
$$

This means that in the case of the presence of spontaneous CP-violation mass of charged Higgs particle is also of order of $\eta$.

We want to stress that the results, we obtained, in the case of the presence of CP-violation are very similar to results obtained in [14 for the case of nonsupersymmetric two Higgs doublet model: for both of cases three neutral and one charged Higgses have masses of order of $\eta$ or less.

7.Let us proceed to numerical investigations of bounds of Higgs boson masses. The Higgs boson masses depend on the parameters $a_{i}, \mathrm{i}=1, \ldots, 8$; $m_{k}^{2}, \mathrm{k}=1, \ldots, 5 ; v_{3} ; \tan \beta ; \varphi ; \alpha$ (for the case of the presence of CP-violation); $\eta=174 \mathrm{GeV}$. The restrictions for $a_{i}, \mathrm{i}=1, \ldots, 8$ are given by formulae $(10)$, (30), (B2), (B3). The parameters $m_{k}^{2}, \mathrm{k}=1, \ldots, 5$ are connected with the supersymmetry breaking and we take them $\sim M_{s}$ or smaller. Besides this, these parameters, as well as $v_{3}$ and $\varphi, \alpha$ are restricted by the conditions (12)-(17),(19),(20) and (33) for the case of the presence of spontaneous CPviolation and also by other conditions connected with positiveness of Higgs boson masses squared. The restrictions for $\tan \beta$ is given by formula (32).

All of the restrictions mentioned above give the bounds for the Higgs boson masses which have been presented in Fig. 1 - 5. These results have been obtained with accuracy of order of $10 \%$.

As we have mentioned above, in the case of absence of CP- violation all Higgs particles besides the lightest one can be as heavy, as supersymmetry breaking scale $M_{s}$. The upper bound for the lightest neutral CP- even Higgs mass $\left(m_{h_{1}}\right)$ as a function of $\tan \beta$ for the cases $M_{s} \sim 100 \mathrm{GeV}, 1000 \mathrm{GeV}$ and $10000 \mathrm{GeV}$ are presented in Fig. 1. As we can see, in this case $m_{h_{1}} \leq$ $105 \mathrm{GeV}, 135 \mathrm{GeV}$ and $150 \mathrm{GeV}$ respectively for $M_{s} \sim 100 \mathrm{GeV}, 1000 \mathrm{GeV}$ and $10000 \mathrm{GeV}$. 
In the case of the presence of CP- violation only two neutral Higgses can be as heavy as supersymmetry breaking scale $M_{s}$. The upper bounds as a functions of $\tan \beta$ we obtained for masses of remaining neutral and charged Higgses $\left(m_{h_{1}}, m_{h_{2}}, m_{h_{3}}\right.$ and $m_{H^{+}}$respectively) are presented in Fig. 2-5. As it follows from Fig.2-5,

$$
\begin{array}{rr}
m_{h_{1}} \leq 30 \mathrm{GeV}, & m_{h_{2}} \leq 95 \mathrm{GeV}, \\
m_{h_{3}} \leq 135 \mathrm{GeV}, & m_{H^{+}} \leq 115 \mathrm{GeV},
\end{array}
$$

for $M_{s} \sim 1000 \mathrm{GeV}$ and

$$
\begin{array}{rr}
m_{h_{1}} \leq 35 \mathrm{GeV}, & m_{h_{2}} \leq 100 \mathrm{GeV}, \\
m_{h_{3}} \leq 150 \mathrm{GeV}, & m_{H^{+}} \leq 145 \mathrm{GeV},
\end{array}
$$

for $M_{s} \sim 10000 \mathrm{GeV}$.

The experimental restrictions for Higgs boson masses in supersymmetric standard model Higgses are the following [26]:

$$
m_{h_{1}}>45 G e V, \quad m_{H^{+}}>45 G e V
$$

There is an obvious contradiction between obtained bound (36) for $m_{h_{1}}$ and the experimental restriction (37). But it is necessary to note that the NMSSM includes additional $S U(2) \times U(1)$ singlets in Higgs sector in compare with the MSSM and so the experimental bounds (37) in general are not true for NMSSM neutral Higgs sector. More precisely, to avoid the contradiction with experiment the lightest neutral Higgs $h_{1}$ must be almost singlet to escape the detection. The preliminary analysis shows that there exists a region of parameter space where such a situation can take place. As we have mentioned above in this case three detectable (i.e. nonsinglet) particles with mass of order of $\eta$ or smaller is always exist.

8.Thus we have investigated bounds for Higgs boson masses in NMSSM which arise from renormalization group equations and scalar Higgs potential analysis. For the case of absence of spontaneous CP-violation we obtain that neutral CP- even Higgs particle with mass smaller than $\eta$ exists. The above restriction is very close to one obtained in MSSM. We have shown that if $M_{s} \sim 100 \mathrm{GeV}$, spontaneous CP-violations isn't possible. If supersymmetry is broken at higher scales, then the scenario with spontaneous CP-violation to be real the lightest neutral Higgs must be almost $S U(2) \times U(1)$ singlet . 
In this case charged Higgs boson have mass smaller than $\eta$ and at least three detectable (i.e. nonsinglet) neutral Higgs boson exist with masses of order of $\eta$. Thus the restrictions obtained for the case of the presence of spontaneous CP- violation are much stronger than in MSSM.

So our analysis of the problem of Higgs masses in NMSSM with the spontaneous CP-breaking shows that the considering model leads to the predictions for Higgs particles masses which can be verified experimentally in the near future. This model can be considered as an interesting alternative of MSSM. The investigation of properties of the considered model in more detail is required. In particular, the correlation between Higgs masses and decay rates in the case, when some of the neutral Higgses are nearly singlets must be investigated. This work is in progress now.

The research described in this publication was made possible in part by Grant N MVU000 from the International Science Foundation.

\section{APPENDIX A}

The $5 \times 5$ symmetric mass matrix $M_{i j}^{2}(\mathrm{i}, \mathrm{j}=1, \ldots, 5)$ of neutral Higgs particles $\Phi_{1}, \Phi_{2}, A, N_{1}, N_{2}$ for the case of the presence CP-violation is given by:

$$
\begin{aligned}
& M_{11}^{2}=\left(\frac{1}{2}\left(a_{1}+a_{2}-2\left(a_{3}+a_{4}\right)\right) \cos ^{2} 2 \beta-\left(a_{2}-a_{1}\right) \cos 2 \beta+\left(a_{1}+a_{2}+2\left(a_{3}+a_{4}\right)\right)\right) \eta^{2} \\
& M_{12}^{2}=\left(-\frac{1}{2}\left(a_{1}+a_{2}-2\left(a_{3}+a_{4}\right)\right) \cos 2 \beta \sin 2 \beta+\frac{1}{2}\left(a_{2}-a_{1}\right) \sin 2 \beta\right) \eta^{2} \\
& M_{13}^{2}=0 \\
& M_{14}^{2}=\left(a_{5}+a_{6}+\left(a_{5}-a_{6}\right) \cos 2 \beta+a_{7} \sin 2 \beta\left(\cos (\varphi-2 \alpha)+\frac{\sin 3 \alpha}{\sin (\varphi+\alpha)}\right)\right) v_{3} \eta \\
& M_{15}^{2}=3 a_{7} v_{3} \eta \sin 2 \beta \sin (\varphi-2 \alpha) \\
& M_{22}^{2}=\frac{1}{2}\left(a_{1}+a_{2}-2\left(a_{3}+a_{4}\right)\right) \eta^{2} \sin ^{2} 2 \beta-\frac{2 a_{7} v_{3}^{2} \sin 3 \alpha}{\sin 2 \beta \sin (\varphi+\alpha)} \\
& M_{23}^{2}=0
\end{aligned}
$$




$$
\begin{aligned}
& M_{24}^{2}=\left(\left(a_{6}-a_{5}\right) \sin 2 \beta+a_{7} \cos 2 \beta\left(\cos (\varphi-2 \alpha)+\frac{\sin 3 \alpha}{\sin (\varphi+\alpha)}\right)\right) v_{3} \eta \\
& M_{25}^{2}=3 a_{7} v_{3} \eta \cos 2 \beta \sin (\varphi-2 \alpha) \\
& M_{33}^{2}=-\frac{2 a_{7} v_{3}^{2} \sin 3 \alpha}{\sin 2 \beta \sin (\varphi+\alpha)} \\
& M_{34}=-a_{7} v_{3} \eta \sin (\varphi-2 \alpha) \\
& M_{35}^{2}=a_{7} v_{3} \eta\left(3 \cos (\varphi-2 \alpha)-\frac{\sin 3 \alpha}{\sin (\varphi+\alpha)}\right) \\
& M_{44}^{2}=4 a_{8} v_{3}^{2}+\frac{a_{7} \eta^{2} \sin 2 \beta \sin (\varphi-2 \alpha)}{2}(3 \cot 3 \alpha+\cot (\varphi+\alpha)) \\
& M_{45}^{2}=-2 a_{7} \eta^{2} \sin 2 \beta \sin (\varphi-2 \alpha) \\
& M_{55}^{2}=-\frac{a_{7} \eta^{2} \sin 2 \beta}{2}\left(9 \sin (\varphi-2 \alpha) \cot 3 \alpha+3 \cos (\varphi-2 \alpha)+\frac{\sin 3 \alpha}{\sin (\varphi+\alpha)}\right)
\end{aligned}
$$

where $\eta^{2}=v_{1}^{2}+v_{2}^{2}=(174 G e V)^{2}$.

For the case of the absence of $\mathrm{CP}$ - violation the $3 \times 3$ symmetric mass matrix $\left(M_{s c}^{2}\right)_{i j}(\mathrm{i}, \mathrm{j}=1,2,3)$ for the scalar $\Phi_{1}, \Phi_{2}, N_{1}$ fields is:

$$
\begin{aligned}
& \left(M_{s c}^{2}\right)_{11}=\left(\frac{1}{2}\left(a_{1}+a_{2}-2\left(a_{3}+a_{4}\right)\right) \cos ^{2} 2 \beta-\left(a_{2}-a_{1}\right) \cos 2 \beta+\left(a_{1}+a_{2}+2\left(a_{3}+a_{4}\right)\right)\right) \eta^{2} \\
& \left(M_{s c}^{2}\right)_{12}=\left(-\frac{1}{2}\left(a_{1}+a_{2}-2\left(a_{3}+a_{4}\right)\right) \cos 2 \beta \sin 2 \beta+\frac{1}{2}\left(a_{2}-a_{1}\right) \sin 2 \beta\right) \eta^{2} \\
& \left(M_{s c}^{2}\right)_{13}=\left(a_{5}+a_{6}+\left(a_{5}-a_{6}\right) \cos 2 \beta\right) v_{3} \eta+\left(m_{4}+2 a_{7} v_{3}\right) \eta \sin 2 \beta \\
& \left(M_{s c}^{2}\right)_{22}=\frac{1}{2}\left(a_{1}+a_{2}-2\left(a_{3}+a_{4}\right)\right) \eta^{2} \sin ^{2} 2 \beta-\frac{2\left(m_{4}+a_{7} v_{3}\right) v_{3}}{\sin 2 \beta} \\
& \left(M_{s c}^{2}\right)_{23}=\left(a_{6}-a_{5}\right) \sin 2 \beta v_{3} \eta-\left(m_{4}+2 a_{7} v_{3}\right) \eta \cos 2 \beta \\
& \left(M_{s c}^{2}\right)_{33}=4 a_{8} v_{3}^{2}+m_{5} v_{3}-\frac{m_{4} \eta^{2} \sin 2 \beta}{2 v_{3}}
\end{aligned}
$$

The $2 \times 2$ symmetric mass matrix $\left(M_{p}^{2}\right)_{i j}(\mathrm{i}, \mathrm{j}=1,2)$ for the pseudoscalar fields $A, N_{2}$ is as follows:

$$
\begin{aligned}
& \left(M_{p}^{2}\right)_{11}=-\frac{2\left(m_{4}+a_{7} v_{3}\right) v_{3}}{\sin 2 \beta} \\
& \left(M_{p}^{2}\right)_{12}=-\left(m_{4}-2 a_{7} v_{3}\right) \eta \\
& \left(M_{p}^{2}\right)_{22}=-3 m_{5} v_{3}-\left(m_{4}+4 a_{7} v_{3}\right) \frac{\eta^{2} \sin 2 \beta}{2 v_{3}}
\end{aligned}
$$




\section{APPENDIX B}

The expressions for $f_{i}, \mathrm{i}=1, \ldots, 8$ in (27) are given by:

$$
\begin{aligned}
f_{1}= & 12 a_{1}^{2}+4 a_{3}^{2}+4 a_{3} a_{4}+2 a_{4}^{2}+2 a_{5}^{2}- \\
& a_{1}\left(3 g_{1}^{2}+9 g_{2}^{2}\right)+\frac{3}{4} g_{1}^{4}+\frac{9}{4} g_{2}^{4}+\frac{3}{2} g_{1}^{2} g_{2}^{2}+12 a_{1} h_{b}^{2}-12 h_{b}^{4} \\
f_{2}= & 12 a_{2}^{2}+4 a_{3}^{2}+4 a_{3} a_{4}+2 a_{4}^{2}+2 a_{6}^{2}- \\
& a_{2}\left(3 g_{1}^{2}+9 g_{2}^{2}\right)+\frac{3}{4} g_{1}^{4}+\frac{9}{4} g_{2}^{4}+\frac{3}{2} g_{1}^{2} g_{2}^{2}+12 a_{2} h_{t}^{2}-12 h_{t}^{4} \\
f_{3}= & 2\left(a_{1}+a_{2}\right)\left(3 a_{3}+a_{4}\right)+4 a_{3}^{2}+2 a_{4}^{2}+2 a_{5} a_{6}-a_{3}\left(3 g_{1}^{2}+9 g_{2}^{2}\right) \\
& +\frac{3}{4} g_{1}^{4}+\frac{9}{4} g_{2}^{4}-\frac{3}{2} g_{1}^{2} g_{2}^{2}+6 a_{3}\left(h_{t}^{2}+h_{b}^{2}\right)-12 h_{t}^{2} h_{b}^{2} \\
f_{4}= & 2 a_{4}\left(a_{1}+a_{2}+4 a_{3}+2 a_{4}\right)+4 a_{7}^{2}- \\
& a_{4}\left(3 g_{1}^{2}+9 g_{2}^{2}\right)+3 g_{1}^{2} g_{2}^{2}+6 a_{4}\left(h_{t}^{2}+h_{b}^{2}\right)+12 h_{t}^{2} h_{b}^{2} \\
f_{5}= & 2 a_{5}\left(3 a_{1}+2 a_{5}+4 a_{8}\right)+2 a_{6}\left(2 a_{3}+a_{4}\right)+ \\
& 8 a_{7}^{2}-\frac{1}{2} a_{5}\left(3 g_{1}^{2}+9 g_{2}^{2}\right)+6 a_{5} h_{b}^{2} \\
f_{6}= & 2 a_{6}\left(3 a_{1}+2 a_{6}+4 a_{8}\right)+2 a_{5}\left(2 a_{3}+a_{4}\right)+ \\
& 8 a_{7}^{2}-\frac{1}{2} a_{6}\left(3 g_{1}^{2}+9 g_{2}^{2}\right)+6 a_{6} h_{t}^{2} \\
f_{7}= & 2 a_{7}\left(a_{3}+2 a_{4}+2 a_{5}+2 a_{6}+2 a_{8}\right) \\
& -\frac{1}{2} a_{7}\left(3 g_{1}^{2}+9 g_{2}^{2}\right)+3 a_{7}\left(h_{t}^{2}+h_{b}^{2}\right) \\
f_{8}= & 2 a_{5}^{2}+2 a_{6}^{2}+4 a_{7}^{2}+20 a_{8}^{2}
\end{aligned}
$$

For $M_{s}=1000 \mathrm{GeV}$ one obtains the following bound for coupling constants $a_{i}$, $\mathrm{i}=1, \ldots, 8$

$$
\begin{array}{r}
0.13 \leq a_{1} \leq 0.24 \quad 0.23 \leq a_{2} \leq 0.3 \quad 0.06 \leq a_{3} \leq 0.19 \\
-0.31 \leq a_{4} \leq 0.15 \quad a_{5} \leq 0.36 \quad a_{6} \leq 0.33 \\
\left|a_{7}\right| \leq 0.19 \quad a_{8} \leq 0.33 \quad 1 \leq\left|h_{t}\right| \leq 1.1 \quad\left|h_{b}\right| \leq 1
\end{array}
$$


And for $M_{s}=10000 \mathrm{GeV}$ :

$$
\begin{array}{r}
0.12 \leq a_{1} \leq 0.35 \quad 0.34 \leq a_{2} \leq 0.35 \quad 0.03 \leq a_{3} \leq 0.31 \\
-0.43 \leq a_{4} \leq 0.26 \quad a_{5} \leq 0.46 \quad a_{6} \leq 0.40 \\
\left|a_{7}\right| \leq 0.22 \quad a_{8} \leq 0.3 \quad 1.0 \leq\left|h_{t}\right|=1.1 \quad h_{b} \leq 1.1
\end{array}
$$




\section{References}

[1] H. P. Nilles, M. Srednicki, D. Willer Phys. Lett. 120B, 366 (1983).

[2] A. Pomarol, Phys. Lett B287, 331 (1992).

[3] A. Pomarol, Phys. Rev., 47D, 273 (1993).

[4] S. Bertolini, F. Visssani, Phys. Lett. B324, 164 (1994).

[5] A. Soni, R. M. Xu, Phys. Rev. Lett. 69, 33 (1992).

[6] T. Elliot, S. King, P. White, Phys. Lett. 305B, 71 (1993).

[7] Y. Okado, Preprint KEK-TH-382 (1993).

[8] U. Ellwanger et al. Preprint LPTHE Orsay 95-04 (hep-ph 9502206).

[9] J. Ellis et al. Phys. Rev. D39, 844 (1989).

[10] L. Maiani et al. Nucl. Phys. B136, 115 (1978).

[11] N. Cabibbo et al. Nucl. Phys. B158, 295 (1979).

[12] B. Pendlton, G. Ross, Phys. Lett. 98B, 221 (1981).

[13] S. Dimopoulos, Proceedings of the XXVII Intern. Conf. on HEP (Glasgow, 1994), p. 93.

[14] H. Asatrian, A. Ioannisyan, S. Matiyan, Z. Phys. C61, 265 (1994).

[15] H. P. Nilles, Preprint MPI-Ph/93-84 (1993).

[16] N. Nilles, Phys. Rep. 110, 1 (1984).

[17] R. Barbiery, S. Ferrara, C. Savoy, Phys. Lett. 119B, 343 (1982).

[18] P. Nath, R. Arnowitt, A. Chamseddine, Phys. Rev. Lett. 49B, 970 (1982)

[19] L. Ibanez, Phys. Lett. 118B, 73 (1982).

[20] L. Ibanez, Nucl. Phys. 218B, 514 (1983). 
[21] S. Soni, H. Weldon, Phys. Lett., 216B, 215 (1983).

[22] P.H.Chankowski,S.Sikorski,J.Rosiek Phys. Lett 281B 100 (1992)

[23] S. P. Martin, M. T. Vaughn, Preprint NUB-3081 93T (hep-ph 9311340).

[24] H.B.Jensen Proceedings of the XXVII Intern. Conf. on HEP (Glasgow, 1994), p. 3.

[25] M. Bando et al. Mod. Phys. Lett. A7, 3379 (1992).

[26] M. Pohl, Proceedings of the XXVII Intern. Conf. on HEP (Glasgow, 1994), p. 107. 
Fig. 1. Bounds on mass of the lightest netral Higgs in the case of absence

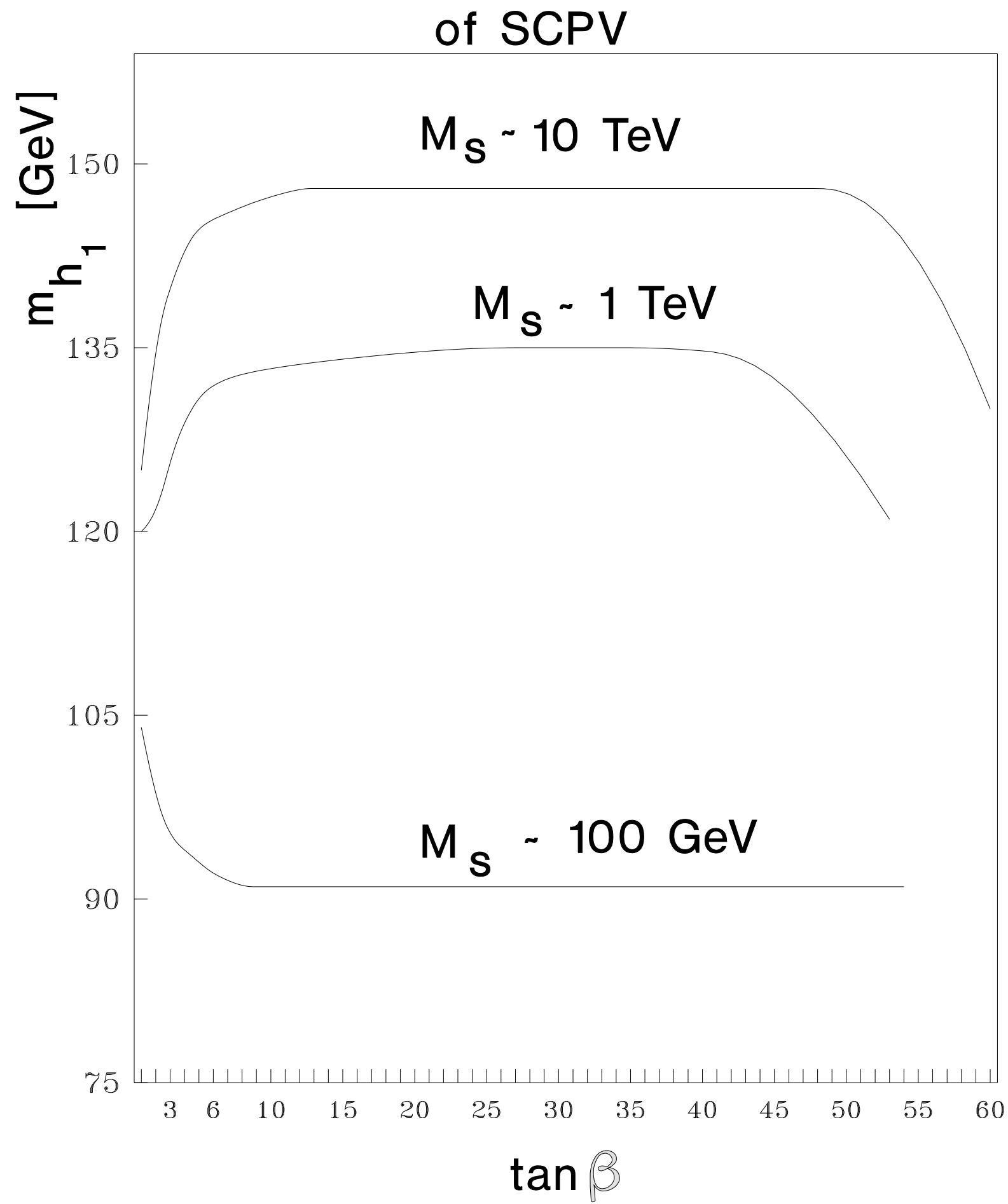


Fig. 2. Bounds on mass of the lightest neutral Higgs in the case of the presence of SCPV

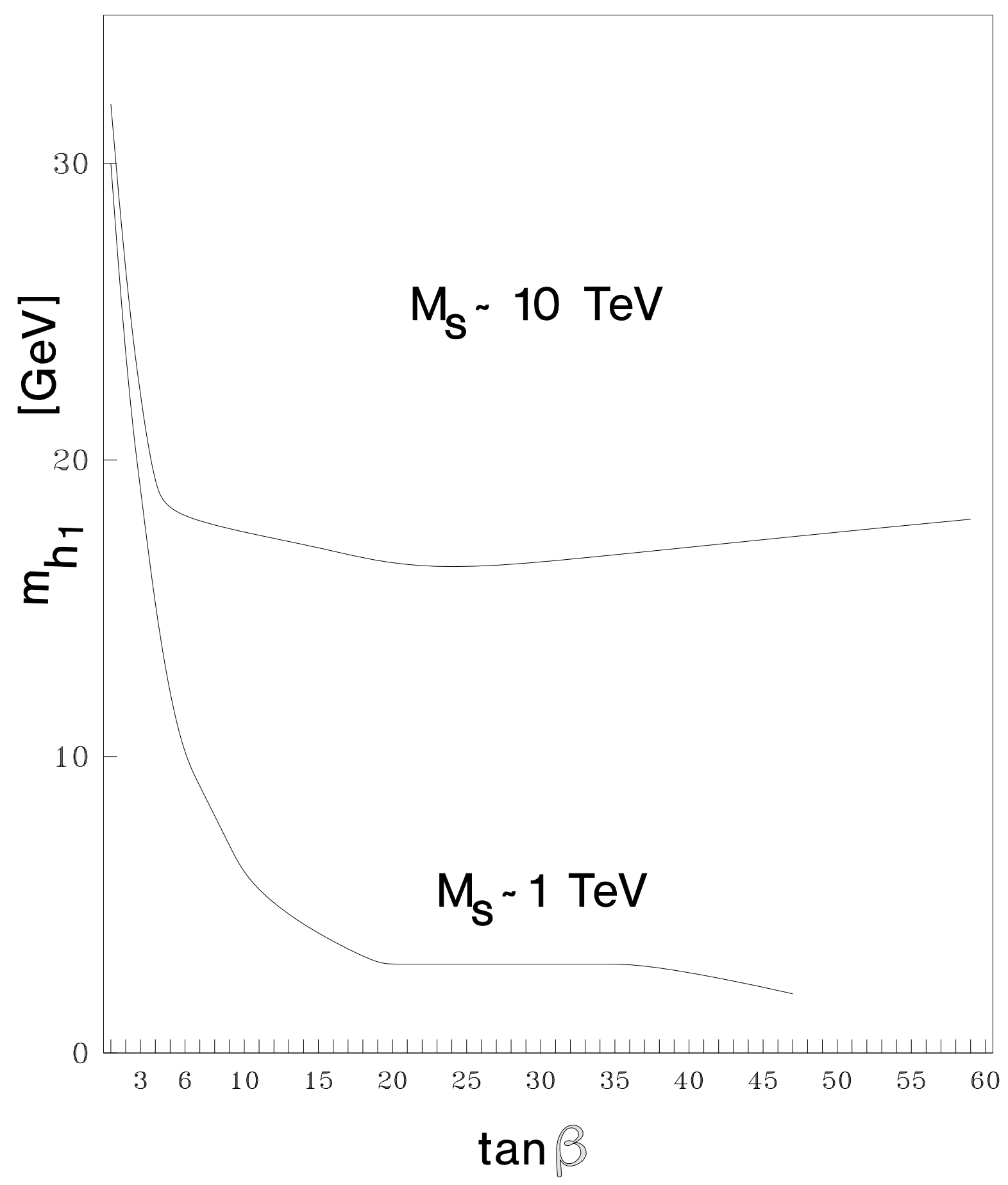


Fig. 3. Bounds on mass of the second lightest neutral Higgs in the case of the presence of SCPV

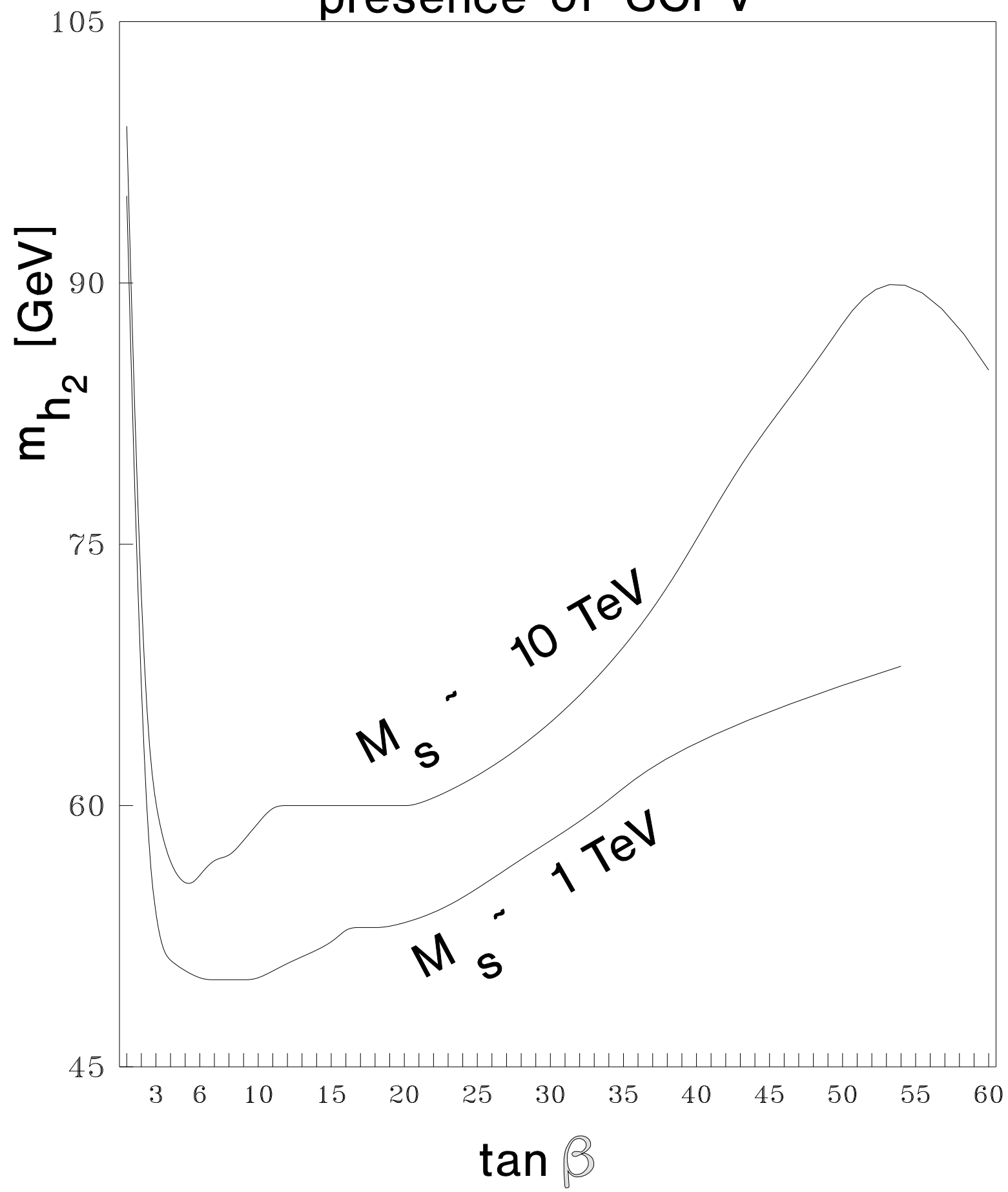


Fig. 4. Bounds on mass of third lightest neutral Higgs in the case of the presence of SCPV

Ð

$\stackrel{5}{E}^{m}$

$M_{S}-10 \mathrm{TeV}$

$M_{S} \sim 1 \mathrm{TeV}$

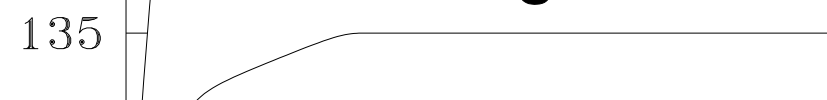


Fig. 5. Bounds on mass of charged Higgs in the case of the presence of SCPV

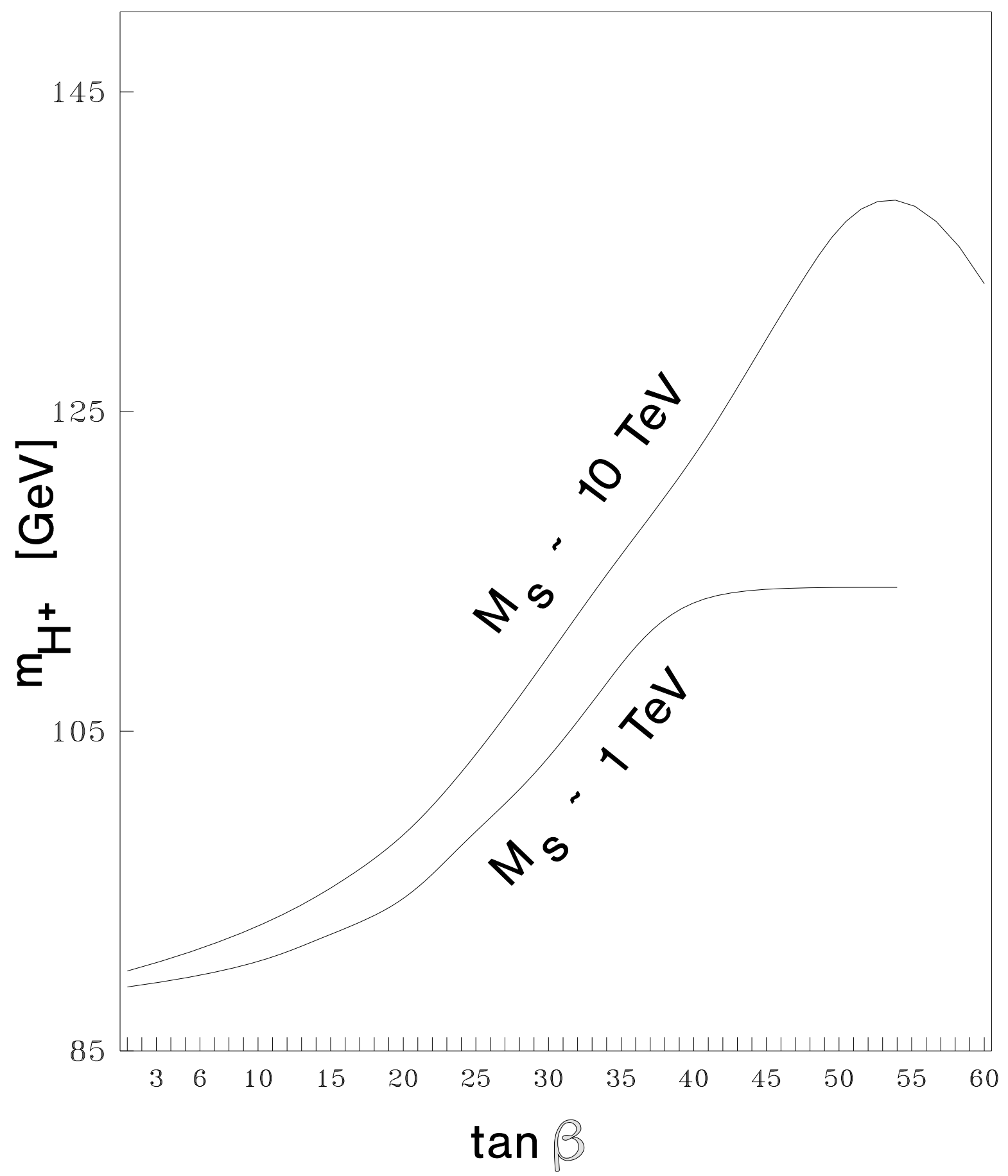

